\title{
Association of PCSK9 with human plasma Lipoproteins
}

\author{
Aikaterini N. Tsouka, Alexandros D. Tselepis \\ Atherothrombosis Research Centre/Laboratory of Biochemistry, Department of Chemistry, University of loannina, \\ loannina, Greece
}

\begin{abstract}
Proprotein Convertase Subtilisin/Kexin type 9 (PCSK9) is a serine protease primarily expressed in the liver. The main function of circulating PCSK9 relates to its binding to the low-density lipoprotein receptor (LDL-R) in hepatocytes, increasing its endosomal and lysosomal degradation. This results in the inhibition of LDL-R recycling to the cell surface and therefore in the reduction of the hepatic LDL uptake, leading to the increase in plasma levels of LDL-cholesterol. Several studies have demonstrated that the plasma levels of PCSK9 are correlated with those of the ApoB-containing lipoproteins; LDL, Lp(a) and Triglyceride-Rich Lipoproteins (TRL). Furthermore, it has been shown that PCSK9 binds to the LDL and Lp(a) particles and significantly influences TRL metabolism. By contrast, controversial results exist concerning the association of PCSK9 with High Density Lipoprotein (HDL). In the present review we present existing data on the association of PCSK9 with human plasma lipoprotein particles and its possible pathophysiological role.
\end{abstract}

KEY WORDS: ApoB-lipoproteins, High Density Lipoprotein (HDL), Low-Density Lipoprotein (LDL), Lp(a), PCSK9, Triglyceride-Rich Lipoproteins

\section{INTRODUCTION}

Proprotein Convertase Subtilisin/Kexin type 9 (PCSK9) is a serine protease primarily expressed in the liver and it is also detected in the central nervous system, the intestine and the kidney as well as in various cell types such as endothelium, smooth muscle cells and macrophages ${ }^{1}$. PCSK9 is also found in the cerebrospinal fluid ${ }^{(2)}$ and at the sites of atherosclerotic plaques ${ }^{3}$.

The PCSK9 circulating in plasma is mainly secreted

\section{Corresponding author:}

Prof. Alexandros Tselepis, MD, PhD Atherothrombosis Research Centre Department of Chemistry, University of loannina 45110 loannina, Greece

Tel.: +30 2651008365 , Fax: +30 2651008785

E-mail: atselep@uoi.gr by the liver ${ }^{1}$. The main function of PCSK9 relates to the binding to low-density lipoprotein receptor (LDL-R) in hepatocytes, increasing its endosomal and lysosomal degradation ${ }^{4}$. This leads to the inhibition of LDL-R recycling to the cell surface and therefore to the reduction of LDL hepatic uptake, leading to the elevation of LDL-cholesterol plasma levels, a major risk factor of cardiovascular diseases $(C V D)^{5}$. Several studies have showed that PCSK9 is associated with various types of lipoproteins in human plasma primarily with LDL, VLDL and Lp(a), with controversial data concerning the association of PCSK9 with HDL. Aim of this review is to describe the association of PCSK9 with various lipoprotein particles in human plasma and to provide existing evidence for the possible pathophysiological role of this association.

Submission: 04.03.2021, Acceptance: 21.04.2021 


\section{PCSK9 BIOSYNTHESIS, INTRACELLULAR METABOLISM, SECRETION AND PATHOPHYSIOLOGICAL ROLE}

The human PCSK9 gene, is located on chromosome 1 p32.3, which contains 12 exons and is 3,617 bps in length ${ }^{6}$. PCSK9 is synthesized as Pro-PCSK9, which consists of 692 amino acids and has a molecular weight of $\sim 75 \mathrm{kDa}^{7}$. The Pro-PCSK9 is composed as a signal peptide (residues 1 to 30), a pro-domain extended between amino acids 31-152, a catalytic domain (residues 153 to 421) and a Cys-His-rich C-terminal domain (CHRD) that extends from 422 to 692 amino acids residues ${ }^{8-10}$. Following its synthesis, Pro-PCSK9 is directed to the Endoplasmic Reticulum (ER) by the signal peptide ${ }^{11}$, where the pro-domain $(\sim 13 \mathrm{kDa})$ is auto-catalytically cleaved off and binds non covalently to the catalytic domain of the remaining PCSK9 protein $(\sim 62 \mathrm{kDa})$. This results in the formation of an intact heterodimer $(62+13 \mathrm{kDa})$ that represents the most active form of the $\mathrm{PCSK}^{6}{ }^{6}$, which is then secreted into the bloodstream through the $\mathrm{ER}^{12,13}$. The $62 \mathrm{kDa}$ PCSK9 can also be proteolytically cleaved in the extracellular space, by protease furin ${ }^{14}$. This cleavage results in the production of a $\sim 7 \mathrm{kDa}$ peptide and the furin-cleaved form (55kDa), of PCSK9 in plasma ${ }^{14}$.

The plasma levels reported by different groups, depending on the methodology used, were $50-600 \mathrm{ng} / \mathrm{ml}$ with a mean value of $200 \mathrm{ng} / \mathrm{ml}^{15,16}, 11-115 \mathrm{ng} / \mathrm{ml}^{15,17}$ and $0.1-9.3 \mu \mathrm{g} / \mathrm{ml}$ with a mean value of $4 \mu \mathrm{g} / \mathrm{ml}^{15,18}$. The above values refer to the total PCSK9 (intact and furin-cleaved) in plasma. The most commonly PCSK9 levels used as a reference are $0.3-0.8 \mu \mathrm{g} / \mathrm{ml}^{19-21}$. Currently there is no method to measure separately the levels of intact or furin-cleaved form of PCSK9.

A major pathophysiological role of PCSK9 is the regulation of the LDL-R on the surface of hepatocytes, thus PCSK9 significantly affects the plasma levels of LDL-cholesterol ${ }^{22}$. This role is exerted by both forms of PCSK9 $(62 \mathrm{kDa}$ and $55 \mathrm{kDa})$, but it is more pronounced for the $62 \mathrm{kDa}$ PCSK9 ${ }^{14}$. More specifically, once secreted into the circulation, PCSK9 interacts with the Endothelial Growth Factor-A (EGF-A) domain of the LDL-R. This complex is internalized in endosomes via clathrin-coated pits and the cytosolic adaptor protein, a phosphotyrosine binding protein named as autosomal recessive hypercholesterolemia $(\mathrm{ARH})^{23}$. The binding affinity of PCSK9 with LDL-R is increased in endosomes due to the existing acidic $\mathrm{pH}$, thus the PCSK9 interaction with the receptor is further potentiated. This increase in the binding affinity may be due to the creation of additional binding sites on the receptor, occurring via intramolecular interactions among receptor domains ${ }^{24,25}$. Importantly, the strong association of PCSK9 with the LDL-
$\mathrm{R}$ in the endosome prevents the conformational changes occurring on the receptor in the absence of PCSK9, which allow LDL-R to dissociate from its lipoprotein ligand and recycle back to the cell surface ${ }^{5,24}$. Consequently, the binding of PCSK9 to the LDL-R leads the receptor to the lysosome for degradation. It should be noted that the domain of the LDL-R which binds to PCSK9 is differentiated from the domain that recognizes and binds to LDL. Indeed, elimination of the LDL binding domain on the LDL-R, has no significant effect on the PCSK9 binding to the receptor. This suggests that the association of the PCSK9 with LDL-R occurs independently on the LDL binding. Since a proportion of circulating PSCK9 is associated with LDL particles (this is described below in this review), it remains to be established whether PCSK9 binds to the LDL-R as a free form or as a complex with LDL particles or in both forms ${ }^{5}$.

Besides the binding and degradation of the LDLR, PCSK9 also binds and leads to degradation of other receptors and membrane proteins, including the VLDL receptor $^{26}$, the Apolipoprotein E Receptor 2 (ApoER2) ${ }^{27}$, the differentiation clusters 36 and 81 (CD36 and CD81, respectively) ${ }^{28,29}$, the beta-secretase 1 (BACE 1$)^{30}$ and the epithelial $(\mathrm{NA}+)$ channel $(\mathrm{ENaC})^{31}$.

\section{ASSOCIATION OF PCSK9 WITH LIPOPROTEINS} Association of PCSK9 with LDL

Clinical studies have demonstrated that plasma PCSK9 levels are positively correlated with LDL levels s2-35 $^{32}$ (Table 1), a correlation that could be attributed to the main PCSK9 function which is the induction of the LDL-R degradation. However, it has been suggested that the above correlation could also be attributed to the association of PCSK9 with LDL particles in the circulation. Indeed, studies have demonstrated that approximately $30 \%$ to $40 \%$ of plasma PCSK9 is associated with LDL through a protein-protein interaction with its ApoB100 content $^{36}$ (figure 1 ) and there is evidence that this association occurs within the secretory pathways of hepatocytes ${ }^{37}$. Moreover, the PCSK9 interaction with ApoB containing lipoproteins leads to the suppression of the ApoB degradation through the autophagosome/lysosome pathway ${ }^{37}$. In parallel, studies have demonstrated that the interaction of PCSK9 with ApoB-100 plays an important role in ApoB-100 metabolism, in an LDL-R independent manner ${ }^{37}$. In support of the above results, another study demonstrated that the removal of ApoB100 containing lipoproteins from plasma by apheresis, reduced the levels of PCSK9 in plasma by $50 \%$. Using immunoblot analysis, this study also showed that the intact form of PCSK9 (62kDa) is bound to LDL whereas 
TABLE 1. In vivo studies demonstrating the correlation of PCSK9 with Lipoproteins

\begin{tabular}{|c|c|c|}
\hline PCSK9/ ApoB & Positive correlation $(r=0.226, p=0.006)$ & \multirow{6}{*}{$\begin{array}{l}\text { Guardiola M, Plana N, Ibarretxe D, Cabré A, } \\
\text { González M, Ribalta J, et al Clin Sci (Lond). } 2015 \\
\text { Jun;128(12):877-82 }\end{array}$} \\
\hline PCSK9/VLDL & Positive correlation $(r=0.210, p=0.001)$ & \\
\hline PCSK9/IDL & Positive correlation $(r=0.206, p=0.001)$ & \\
\hline PCSK9/ Small LDL & Positive correlation $(r=0.224, p<0.001)$ & \\
\hline PCSK9/ Medium LDL & Positive correlation $(r=0.235, p<0.001)$ & \\
\hline PCSK9/Very Small LDL & Positive correlation $(r=0.220, p<0.001)$ & \\
\hline PCSK9/ HDL-Cholesterol & Positive correlation $(r=0.149, p=0.012)$ & \multirow{9}{*}{$\begin{array}{l}\text { Xu RX, Li S, Zhang Y, Li XL, Guo YL, Zhu CG, et al. } \\
\text { Lipids Health Dis. } 2014 \text { Dec 11;13:188. }\end{array}$} \\
\hline PCSK9/ non HDL-Cholesterol & Positive correlation $(r=0.221, p=0.000)$ & \\
\hline PCSK9/ Apo Al & Positive correlation $(r=0.184, p=0.003$ & \\
\hline PCSK9/ LDL-Cholesterol & Positive correlation $(r=0.219, p=0.000)$ & \\
\hline PCSK9/ ApoB & Positive correlation $(r=0.260, p=0.000)$ & \\
\hline PCSK9/ Small LDL & Positive correlation $(r=0.166, p=0.005)$ & \\
\hline PCSK9/ Intermediate LDL & Positive correlation $(r=0.145, p=0.015)$ & \\
\hline PCSK9/ Small HDL & Positive correlation $(r=0.124, p=0.048)$ & \\
\hline PCSK9/ Intermediate HDL & Positive correlation $(r=0.157, p=0.013$ ) & \\
\hline PCSK9/ LDL-Cholesterol & Positive correlation $(r=0.516, p<0.001)$ & \multirow{7}{*}{$\begin{array}{l}\text { Kwakernaak AJ, Lambert G, Dullaart RP Clin } \\
\text { Biochem. } 2014 \text { May;47(7-8):679-82. }\end{array}$} \\
\hline PCSK9/ non HDL-Cholesterol & Positive correlation $(r=0.547, p<0.001)$ & \\
\hline PCSK9/ HDL-Cholesterol & Not correlated $(r=0.063, p=0.66)$ & \\
\hline PCSK9/VLDL & Positive correlation $(r=0.333, p=0.022)$ & \\
\hline PCSK9/ LDL & Positive correlation $(r=0.373, p=0.010)$ & \\
\hline PCSK9/IDL & Positive correlation $(\mathrm{r}=0.532, \mathrm{p}=<0.001)$ & \\
\hline PCSK9/ Small LDL & Positive correlation $(r=0.329, p=0.024)$ & \\
\hline PCSK9/ LDL-Cholesterol & Positive correlation $(p<0.001)$ & \multirow{2}{*}{$\begin{array}{l}\text { Ferri N, Ruscica M, Coggi D, Bonomi A, Amato M, } \\
\text { Frigerio B, et al, Atherosclerosis. } 2020 \text { Sep;309:39-46 }\end{array}$} \\
\hline PCSK9/ HDL-Cholesterol & Positive correlation $(p<0.001)$ & \\
\hline
\end{tabular}

most of the furin-cleaved form is in the ApoB-100-free fraction of plasma ${ }^{38}$.

The interaction between PCSK9 and LDL has also been studied, in vitro ${ }^{39}$. In this regard, LDL isolated by flotation ultracentrifugation, by which the endogenous PCSK9 was completely detached, was incubated in vitro with fluorescently labeled recombinant PCSK9. The association between LDL and PCSK9 was studied using immunoprecipitation and western blot analysis ${ }^{39}$. The results showed that the isolated LDL binds to the PCSK9 ${ }^{39}$. Regarding the PCSK9 domains involved in its binding with $\mathrm{LDL}^{39}$, it has been demonstrated that the $\mathrm{N}$-terminal sequence of the PCSK9 prodomain containing the 31-52 amino acids is required for the binding of PCSK9 with LDL ${ }^{40}$ (figure 1). Interestingly, deletion of this amino acid sequence, prevents the LDL binding to PCSK9, however it increases the PCSK9 binding affinity to the LDL- $\mathrm{R}^{40}$.

Since LDL is consisted of a heterogeneous particle population, it was investigated whether there are differences in the association of PCSK9 with various LDL subfractions. In this regard, it has been shown that the PCSK9 levels are positively and independently correlated with small and intermediate LDL subfractions in plasma of patients with stable CAD ${ }^{33}$. Another study demonstrated that PCSK9 levels are positively correlated with medium, small and very small LDL subfractions, in plasma of patients with high cardiovascular risk ${ }^{34}$. A gender analysis performed in this study showed that this correlation, is observed in male subjects but not in females, suggesting that the differential association of PCSK9 with LDL subfractions may represent a new underlying mechanism for gender disparity in the development of $C A D^{33}$. In conclusion, the PCSK9 levels in plasma are positively correlated with LDL, preferentially with the intermediate, and small LDL particles. The intact form of PCSK9 rather than its the furin-cleaved form is primarily associated LDL through the binding of its $\mathrm{N}$-terminal region of the prodomain to ApoB-100. 


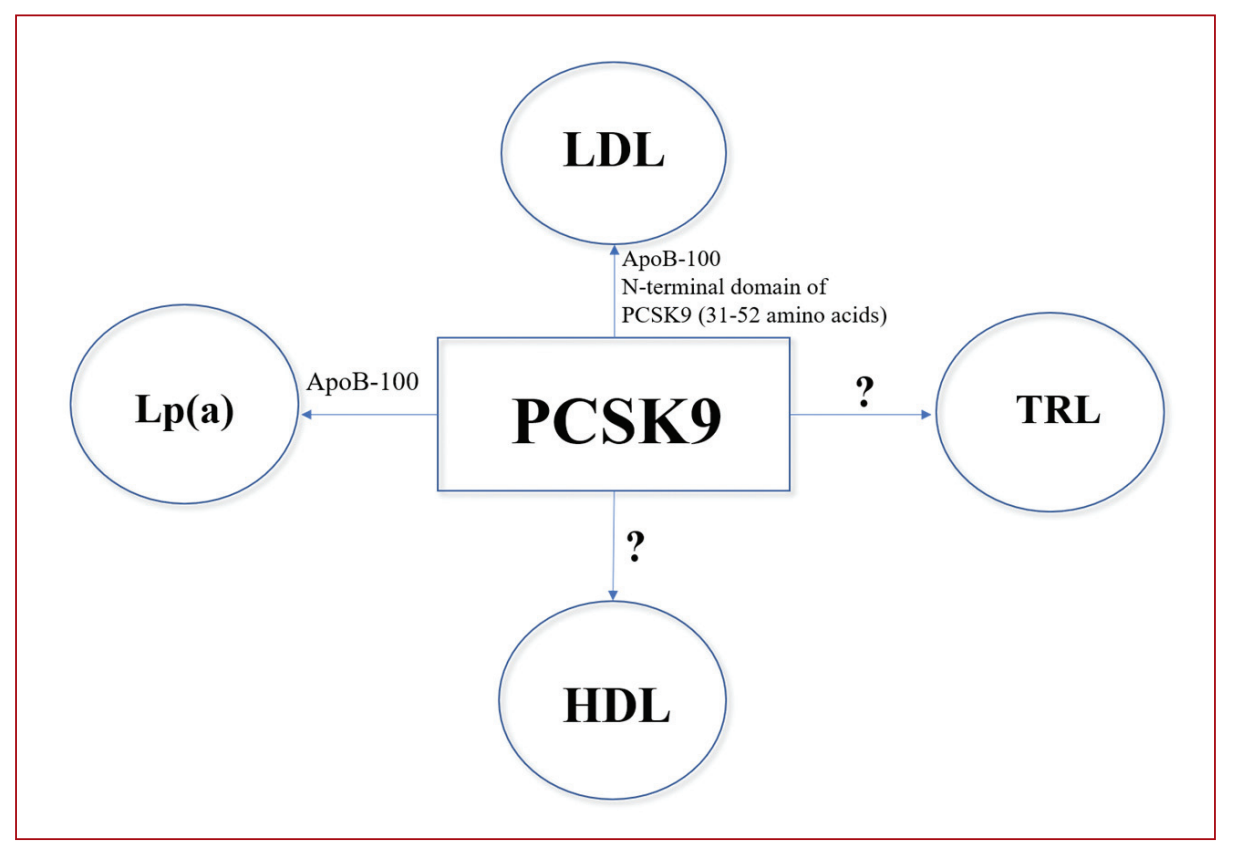

FIGURE 1. Association of PCSK9 with human lipoprotein particles and the domains which play a crucial role in this association.

\section{Association of PCSK9 with Triglyceride-Rich Lipoproteins (TRL)}

Triglyceride-rich lipoproteins (TRL) include chylomicrons (CM), very low density lipoproteins (VLDL) and their remnants ${ }^{41}$. Studies have demonstrated a positive correlation between circulating PCSK9 and plasma triglyceride levels ${ }^{32-34}$. This correlation was the first indication that PCSK9 may play a role in TRL metabolism ${ }^{42}$. Previously published data have demonstrated that the reduction in plasma PCSK9 levels is associated with a decrease in TG levels, possibly through an enhancement of the hepatic catabolism of intermediate density lipoprotein (IDL) via the LDL-R pathway ${ }^{43}$. Consistent with the above results, is the finding that the plasma PCSK9 levels are positively correlated with IDL and VLDL levels ${ }^{32,34}$ (Table 1). It has been demonstrated that PCSK9 does not bind to isolated VLDL from human plasma ${ }^{39}$, whereas a study of isolated VLDL from mice plasma using Fast Protein Liquid Chromatography (FPLC) interacts with PCSK9 ${ }^{37}$. Another mechanism by which PCSK9 may affect VLDL levels is through its binding to the VLDL receptor, probably through the EGF-A domain in a similar manner as it binds to the LDL- $\mathrm{R}^{44}$. Moreover, increased cellular and secreted ApoB-48 and ApoB-100 levels have been shown after treatment of human enterocytes with recombinant PCSK9, indicating that PCSK9 may induce the production of $\mathrm{CM}$ in the intestinal cells ${ }^{41}$. In addition, incubation of this enterocyte cell line with a human Gain of Function D374Y-PCSK9 had as a consequence an enhanced cholesterol uptake in these cells (due to increased expression of cholesterol transporters NPC1L1 and CD36) and an increased CM secretion (due to increased lipid and ApoB-48 biogenesis) as compared with normal PCSK9 ${ }^{41}$. In conclusion, PCSK9 levels are positively correlated with plasma levels of triglyceride rich lipoproteins, including CM, VLDL and IDL. PCSK9 may influence CM production as well as VLDL and IDL degradation, however the binding of PCSK9 with VLDL particles needs further investigation.

\section{Association of PCSK9 with Lp(a)}

Lp(a) consists of an LDL-like particle on which a unique apolipoprotein (a) is attached, through a single disulfide bond formed with the LDL ApoB-100 moiety ${ }^{45}$. High Lp(a) levels in plasma, are positively correlated with PCSK9 levels, in patients not receiving any lipid lowering drug ${ }^{11}$. Moreover, several studies have demonstrated that in addition to LDL, PCSK9 may bind to Lp(a), since it also contains ApoB-10011,38 (figure 1). To establish the association of PCSK9 with $\operatorname{Lp}(\mathrm{a})$, investigators isolated $L D L$ and $L p(a)$ from subjects with elevated levels of $L p(a)$, using iodixanol-based ultracentrifugation. The possible association of PCSK9 with Lp(a) was investigated by native gel electrophoresis and immunoblotting. Using these techniques authors showed that the $L p(a)$ band contained ApoB, Apo(a) and also PCSK9, strengthening the notion that PCSK9 associates with $\operatorname{Lp}(a)^{38}$. The PCSK9 bound on Lp(a) consisted of both the intact and the furin-cleaved form ${ }^{11}$. Finally, it has been suggested that PCSK9 could preferentially be associated with Lp(a) 
as compared with LDL, due to longer Lp(a) half-life in human plasma ${ }^{11,46}$.

\section{Is PCSK9 associated with High Density Lipoprotein?}

Some clinical studies have demonstrated that the PCSK9 levels in plasma are positively correlated with HDL levels ${ }^{33,35}$, whereas others failed to show such correlation $^{32}$ (Table 1 ). In addition, there are contrasting results as concern the association of PCSK9 with HDL particles. Previous data have shown that PCSK9 is associated with apoB-containing lipoproteins such as LDL and Lp(a) but not with $\mathrm{HDL}^{39}$. In contrast, a more recent study suggests that the main carrier of PCSK9 in plasma are the HDL particles ${ }^{47}$. By using plasma from healthy volunteers, this study demonstrated that approximately $20 \%$ of total plasma PCSK9 was immunoprecipitated with a specific anti-ApoB antibody which binds to all apoB-containing lipoproteins, including $\mathrm{Lp}(\mathrm{a})^{47}$. Importantly, removal of HDL from plasma by immunoprecipitation, using a specific anti-ApoA1 antibody, resulted in an approximately $90 \%$ reduction of total PCSK9 plasma levels ${ }^{47}$. The association of PCSK9 with HDL in this study was further supported by the finding that immunoblotting of total plasma, HDL-depleted plasma and HDL particles prepared from plasma. Results showed that HDL particles contain both intact and furin-cleaved PCSK9 forms. In this regard it should be emphasized that immunoisolation of HDL, rather than isolation by sequential ultracentrifugation of plasma, may reduce the loss of HDL-associated PCSK9, since studies have shown that the high $\mathrm{g}$ forces required for HDL isolation by ultracentrifugation, may result in dissociation of PCSK9 from HDL particles ${ }^{39}$. Based on the above controversial results on whether PCSK9 is associated with HDL, it is concluded that the possible association of PCSK9 with HDL particle needs further investigation.

\section{Acknowledgment}

The present study was supported by a research grant from the Hellenic Atherosclerosis Society which was received by Tsouka N. Aikaterini.

\section{Conflict of interest}

The authors have no conflicts of interest related to this publication.

\title{
ПЕРІАНЧH
}

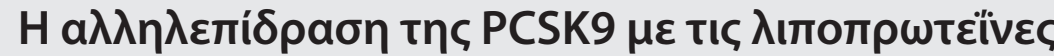

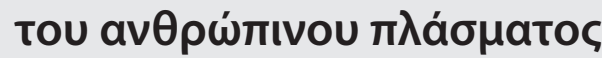

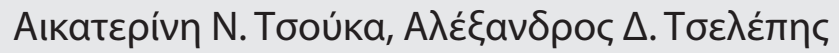

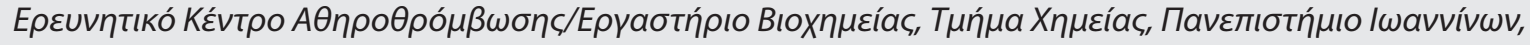 \\ I $\omega a ́ v v i v a, E \lambda \lambda a ́ \delta a$
}

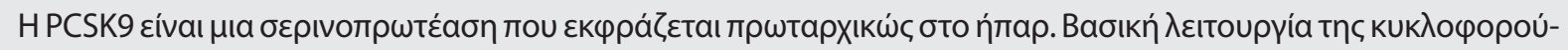

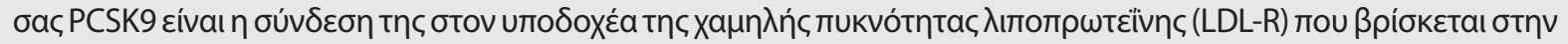

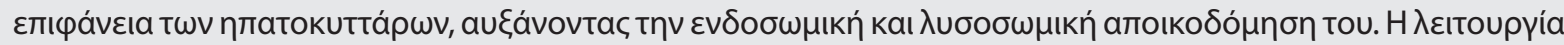

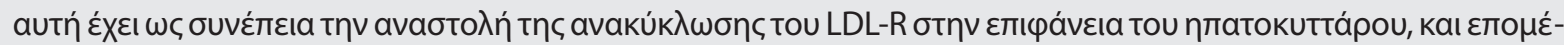

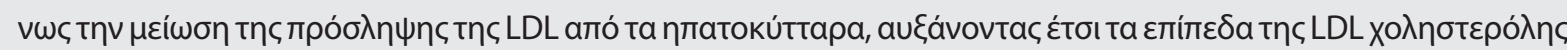

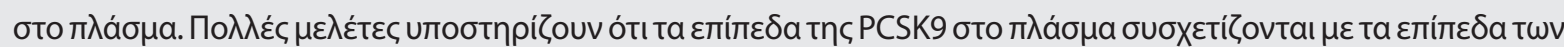

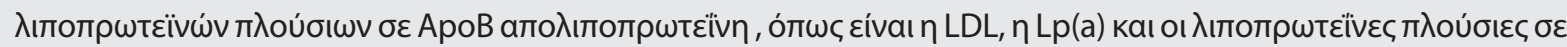

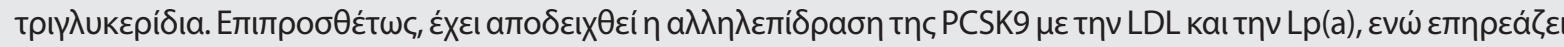

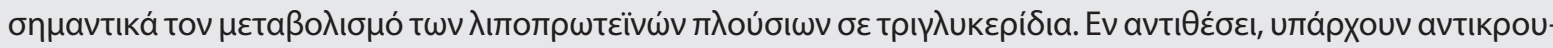

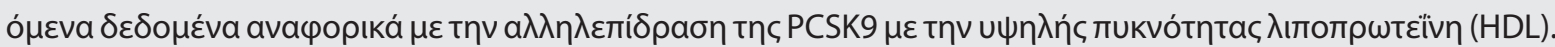

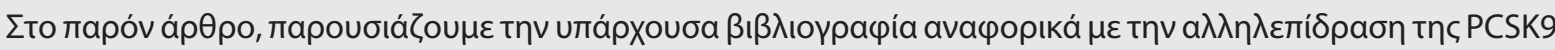
$\mu \varepsilon$ та $\lambda$ เтопр

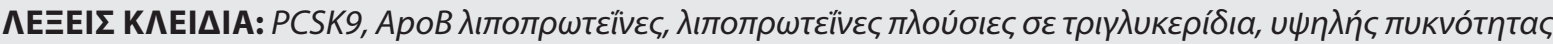

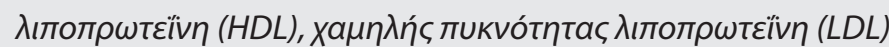




\section{REFERENCES}

1. Norata GD, Tavori H, Pirillo A, Fazio S, Catapano AL. Biology of proprotein convertase subtilisin kexin 9: Beyond low-density lipoprotein cholesterol lowering. Cardiovasc Res [Internet]. 2016 Oct; 112(1):429-42. Available from: https://www.ncbi.nlm.nih.gov/pubmed/27496869.

2. Chen YQ, Troutt JS, Konrad RJ. PCSK9 is present in human cerebrospinal fluid and is maintained at remarkably constant concentrations throughout the course of the day. Lipids [Internet]. 2014 May;49(5):445-55. Available from: https://www.ncbi.nlm.nih.gov/pubmed/24659111.

3. Ferri N, Tibolla G, Pirillo A, Cipollone F, Mezzetti A, Pacia $S$, et al. Proprotein convertase subtilisin kexin type 9 (PCSK9) secreted by cultured smooth muscle cells reduces macrophages LDLR levels. Atherosclerosis [Internet]. 2012 Feb;220(2):381-6. Available from: https://www.ncbi.nlm. nih.gov/pubmed/22176652.

4. Schulz R, Schlüter KD. PCSK9 targets important for lipid metabolism. Clin Res Cardiol Suppl. 2017 Mar;12(Suppl 1):2-11.

5. Zhang DW, Lagace TA, Garuti R, Zhao Z, McDonald M, Horton JD, et al. Binding of proprotein convertase subtilisin/kexin type 9 to epidermal growth factor-like repeat A of low density lipoprotein receptor decreases receptor recycling and increases degradation. J Biol Chem [Internet]. 2007 Jun;282(25):18602-12. Available from: https:// www.ncbi.nlm.nih.gov/pubmed/17452316.

6. Tsouka AN, Tellis CC, Tselepis AD. Pharmacology of PCSK9 Inhibitors: Current status and future perspectives. Curr Pharm Des [Internet]. 2018;24(31):3622-33. Available from: https://www.ncbi.nlm.nih.gov/pubmed/30317986.

7. Shapiro MD, Tavori H, Fazio S. PCSK9: From basic science discoveries to clinical trials. Circ Res [Internet]. 2018 May;122(10):1420-38. Available from: https://www.ncbi. nlm.nih.gov/pubmed/29748367.

8. Cunningham D, Danley DE, Geoghegan KF, Griffor MC, Hawkins JL, Subashi TA, et al. Structural and biophysical studies of PCSK9 and its mutants linked to familial hypercholesterolemia. Nat Struct Mol Biol [Internet]. 2007 May;14(5):413-9. Available from: https://www.ncbi.nlm. nih.gov/pubmed/17435765.

9. Hampton EN, Knuth MW, Li J, Harris JL, Lesley SA, Spraggon $\mathrm{G}$. The self-inhibited structure of full-length PCSK9 at 1.9 A reveals structural homology with resistin within the C-terminal domain. Proc Natl Acad Sci U S A [Internet]. 2007 Sep; 104(37):14604-9. Available from: https://www. ncbi.nlm.nih.gov/pubmed/17804797.

10. Piper DE, Jackson S, Liu Q, Romanow WG, Shetterly S, Thibault ST, et al. The crystal structure of PCSK9: a regulator of plasma LDL-cholesterol. Structure [Internet]. 2007 May;15(5):545-52. Available from: https://www.ncbi.nlm. nih.gov/pubmed/17502100.

11. Tavori H, Christian D, Minnier J, Plubell D, Shapiro MD, Yeang C, et al. PCSK9 Association with lipoprotein(a). Circ Res [Internet]. 2016 Jun;1 19(1):29-35. Available from: https://www.ncbi.nlm.nih.gov/pubmed/27121620.

12. Benjannet $S$, Rhainds D, Essalmani R, Mayne J, Wickham L, Jin W, et al. NARC-1/PCSK9 and its natural mutants: zymogen cleavage and effects on the low density lipoprotein (LDL) receptor and LDL cholesterol. J Biol Chem
[Internet]. 2004 Nov;279(47):48865-75. Available from: https://www.ncbi.nlm.nih.gov/pubmed/15358785.

13. Maxwell KN, Breslow JL. Adenoviral-mediated expression of Pcsk9 in mice results in a low-density lipoprotein receptor knockout phenotype. Proc Natl Acad Sci U S A [Internet]. 2004 May;101(18):7100-5. Available from: https://www.ncbi.nlm.nih.gov/pubmed/15118091.

14. Oleaga C, Hay J, Gurcan E, David LL, Mueller PA, Tavori $\mathrm{H}$, et al. Insights into the kinetics and dynamics of the furin-cleaved form of PCSK9. J Lipid Res [Internet]. 2021 Jan;62(15):100003. Available from: https://doi. org/10.1194/jlr.RA120000964.

15. Dubuc G, Tremblay M, Paré G, Jacques H, Hamelin J, Benjannet $S$, et al. A new method for measurement of total plasma PCSK9: Clinical applications. J Lipid Res. 2010 Jan;51(1):140-9.

16. Lagace TA, Curtis DE, Garuti R, McNutt MC, Sahng WP, Prather HB, et al. Secreted PCSK9 decreases the number of LDL receptors in hepatocytes and in livers of parabiotic mice. J Clin Invest. 2006 Nov; 116(11):2995-3005.

17. Alborn WE, Cao G, Careskey HE, Qian YW, Subramaniam DR, Davies J, et al. Serum proprotein convertase subtilisin kexin type 9 is correlated directly with serum LDL cholesterol. Clin Chem. 2007 Oct;53(10):1814-9.

18. Lambert G, Ancellin N, Charlton F, Comas D, Pilot J, Keech A, et al. Plasma PCSK9 concentrations correlate with LDL and total cholesterol in diabetic patients and are decreased by fenofibrate treatment. Clin Chem. 2008 Jun;54(6):1038-45.

19. Qi Z, Hu L, Zhang J, Yang W, Liu X, Jia D, et al. PCSK9 (Proprotein Convertase Subtilisin/Kexin 9) Enhances platelet activation, thrombosis, and myocardial infarct expansion by binding to platelet CD36. Circulation. 2021 Jan;143(1):45-61.

20. Ridker PM, Rifai N, Bradwin G, Rose L. Plasma proprotein convertase subtilisin/kexin type 9 levels and the risk of first cardiovascular events. Eur Heart J. 2016 Feb;37(6):554-60.

21. Navarese EP, Kolodziejczak M, Winter MP, Alimohammadi A, Lang IM, Buffon A, et al. Association of PCSK9 with platelet reactivity in patients with acute coronary syndrome treated with prasugrel or ticagrelor: The PCSK9-REACT study. Int J Cardiol [Internet]. 2017 Jan;227:644-9. Available from: http://dx.doi.org/10.1016/j.ijcard.2016.10.084.

22. Melendez QM, Krishnaji ST, Wooten CJ, Lopez D. Hypercholesterolemia:The role of PCSK9. Arch Biochem Biophys [Internet]. 2017 Jul;625-626:39-53. Available from: https:// www.ncbi.nlm.nih.gov/pubmed/28587771.

23. Malito E, Bursulaya B, Chen C, Lo Surdo P, Picchianti M, Balducci E, et al. Structural basis for lack of toxicity of the diphtheria toxin mutant CRM197. Proc Natl Acad Sci U S A [Internet]. 2012 Apr;109(14):5229-34. Available from: https://www.ncbi.nlm.nih.gov/pubmed/22431623.

24. Poirier S, Mayer G. The biology of PCSK9 from the endoplasmic reticulum to lysosomes: new and emerging therapeutics to control low-density lipoprotein cholesterol. Drug Des Devel Ther [Internet]. 2013 Oct;7:113548. Available from: https://www.ncbi.nlm.nih.gov/pubmed/24115837.

25. Yamamoto T, Lu C, Ryan RO. A two-step binding model of PCSK9 interaction with the low density lipoprotein receptor. J Biol Chem. 2011 Feb;286(7):5464-70. 
26. Roubtsova A, Munkonda MN, Awan Z, Marcinkiewicz J, Chamberland A, Lazure C, et al. Circulating proprotein convertase subtilisin/kexin 9 (PCSK9) regulates VLDLR protein and triglyceride accumulation in visceral adipose tissue. Arter Thromb Vasc Biol [Internet]. 2011 Apr;31(4):785-91. Available from: https://www.ncbi.nlm. nih.gov/pubmed/21273557.

27. Roubtsova A, Chamberland A, Marcinkiewicz J, Essalmani $\mathrm{R}$, Fazel A, Bergeron JJ, et al. PCSK9 deficiency unmasks a sex- and tissue-specific subcellular distribution of the LDL and VLDL receptors in mice. J Lipid Res [Internet]. 2015 Nov;56(11):2133-42. Available from: https://www. ncbi.nlm.nih.gov/pubmed/26323289.

28. Demers A, Samami S, Lauzier B, Des Rosiers C, Ngo Sock ET, Ong $\mathrm{H}$, et al. PCSK9 induces CD36 degradation and affects long-chain fatty acid uptake and triglyceride metabolism in adipocytes and in mouse liver. Arter Thromb Vasc Biol [Internet]. 2015 Day;35(12):2517-25. Available from: https://www.ncbi.nlm.nih.gov/pubmed/26494228.

29. Le QT, Blanchet M, Seidah NG, Labonte P. Plasma Membrane Tetraspanin CD81 Complexes with Proprotein Convertase Subtilisin/Kexin Type 9 (PCSK9) and Low Density Lipoprotein Receptor (LDLR), and Its Levels Are Reduced by PCSK9. J Biol Chem [Internet]. 2015 Sep;290(38):23385-400. Available from: https://www. ncbi.nlm.nih.gov/pubmed/26195630.

30. Jonas MC, Costantini C, Puglielli L. PCSK9 is required for the disposal of non-acetylated intermediates of the nascent membrane protein BACE1. EMBO Rep [Internet]. 2008 Sep;9(9):916-22. Available from: https://www.ncbi. nlm.nih.gov/pubmed/18660751.

31. Sharotri V, Collier DM, Olson DR, Zhou R, Snyder PM. Regulation of epithelial sodium channel trafficking by proprotein convertase subtilisin/kexin type 9 (PCSK9). J Biol Chem [Internet]. 2012 Jun;287(23):19266-74. Available from: https://www.ncbi.nlm.nih.gov/pubmed/22493497.

32. Kwakernaak AJ, Lambert G, Dullaart RP. Plasma proprotein convertase subtilisin-kexin type 9 is predominantly related to intermediate density lipoproteins. Clin Biochem [Internet]. 2014 May;47(7-8):679-82. Available from: https://www.ncbi.nlm.nih.gov/pubmed/24680982.

33. Xu RX, Li S, Zhang Y, Li XL, Guo YL, Zhu CG, et al. Relation of plasma PCSK9 levels to lipoprotein subfractions in patients with stable coronary artery disease. Lipids Health Dis. 2014 Dec;13(1):1-9.

34. Guardiola M, Plana N, Ibarretxe D, Cabré A, González M, Ribalta J, et al. Circulating PCSK9 levels are positively correlated with NMR-assessed atherogenic dyslipidaemia in patients with high cardiovascular risk. Clin Sci. 2015 Jun;128(12):877-82.

35. Ferri N, Ruscica M, Coggi D, Bonomi A, Amato M, Frigerio $B$, et al. Sex-specific predictors of PCSK9 levels in a European population: The IMPROVE study. Atherosclerosis [Internet]. 2020 Sep;309(April):39-46. Available from: https://doi.org/10.1016/j.atherosclerosis.2020.07.014.

36. Sarkar SK, Foo ACY, Matyas A, Asikhia I, Kosenko T, Goto $\mathrm{NK}$, et al. A transient amphipathic helix in the prodomain of PCSK9 facilitates binding to low-density lipoprotein particles. J Biol Chem. 2020 Feb;295(8):2285-98.

37. Sun H, Samarghandi A, Zhang N, Yao Z, Xiong M, Teng BB. Proprotein convertase subtilisin/kexin type 9 interacts with apolipoprotein $B$ and prevents its intracellular degradation, irrespective of the low-density lipoprotein receptor. Arter Thromb Vasc Biol [Internet]. 2012 Jul;32(7):1585-95. Available from: https://www.ncbi.nlm. nih.gov/pubmed/22580899.

38. Tavori H, Giunzioni I, Linton MF, Fazio S. Loss of plasma proprotein convertase subtilisin/kexin 9 (PCSK9) after lipoprotein apheresis. Circ Res [Internet]. 2013 Dec;113(12):1290-5. Available from: https://www.ncbi. nlm.nih.gov/pubmed/24122718.

39. Kosenko T, Golder M, Leblond G, Weng W, Lagace TA. Low density lipoprotein binds to proprotein convertase subtilisin/kexin type-9 (PCSK9) in human plasma and inhibits PCSK9-mediated low density lipoprotein receptor degradation. J Biol Chem [Internet]. 2013 Mar;288(12):827988. Available from: https://www.ncbi.nlm.nih.gov/pubmed/23400816.

40. Hyock JK, Lagace TA, McNutt MC, Horton JD, Deisenhofer J. Molecular basis for LDL receptor recognition by PCSK9. Proc Natl Acad Sci U S A. 2008 Feb;105(6):1820-5.

41. Druce I, Abujrad H, Ooi TC. PCSK9 and triglyceride-rich lipoprotein metabolism. J Biomed Res [Internet]. 2015 Jul;29(6):429-36. Available from: https://www.ncbi.nlm. nih.gov/pubmed/26320603.

42. Arsenault BJ, Pelletier-Beaumont E, Almeras N, Tremblay A, Poirier P, Bergeron J, et al. PCSK9 levels in abdominally obese men: association with cardiometabolic risk profile and effects of a one-year lifestyle modification program. Atherosclerosis [Internet]. 2014 Oct;236(2):3216. Available from: https://www.ncbi.nlm.nih.gov/pubmed/25128757.

43. Dijk W, Le May C, Cariou B. Beyond LDL: What Role for PCSK9 in Triglyceride-Rich Lipoprotein Metabolism? Trends Endocrinol Metab [Internet]. 2018 Jun;29(6):420-34. Available from: https://doi.org/10.1016/j.tem.2018.03.013.

44. Shan L, Pang L, Zhang R, Murgolo NJ, Lan H, Hedrick JA. PCSK9 binds to multiple receptors and can be functionally inhibited by an EGF-A peptide. Biochem Biophys Res Commun [Internet]. 2008 Oct;375(1):69-73. Available from: https://www.ncbi.nlm.nih.gov/pubmed/18675252.

45. Tselepis AD. Oxidized phospholipids and lipoproteinassociated phospholipase $A_{2}$ as important determinants of $L p(a)$ functionality and pathophysiological role. J Biomed Res [Internet]. 2018 Jan;31(1):13-22. Available from: https://www.ncbi.nlm.nih.gov/pubmed/27346583.

46. Rader DJ, Mann WA, Cain W, Kraft HG, Usher D, Zech LA, et al. The low density lipoprotein receptor is not required for normal catabolism of $L p(a)$ in humans. J Clin Invest [Internet]. 1995 Mar;95(3):1403-8. Available from: https:// www.ncbi.nlm.nih.gov/pubmed/7883987.

47. Burnap SA, Joshi A, Tsimikas S, Fernández-Hernando C, Kiechl S, Berry SE, et al. High-Density lipoproteins are the main carriers of pcsk9 in the circulation. J Am Coll Cardiol [Internet]. 2020 Mar;75(12):1495-7. Available from: https://doi.org/10.1016/j.jacc.2020.01.033. 